\title{
Considerations about energy consumption in sensor nodes over wireless personal area networks using MiWi protocol
}

Gustavo Meneses Benavides, M.Sc. / gustavo.meneses@usbmed.edu.co

Universidad San Buenaventura, Medellín, Colombia

ABSTRACT Adopting techniques to increase operation time of battery-powered sensor nodes in wireless networks is necessary when implementing practical monitoring applications intended to run over extended periods of time. Energy harvesting; DC-DC converter techniques; and features of modern microcontrollers like extreme lower consumption, Sleep and Idle states, can contribute to improve the performance of network nodes. Designers can also use the possibilities offered by special protocols for wireless sensor networks, routing algorithms, and data aggregation plus collection strategies. Issues related to the process of design and implementation of an energy-efficient sensor node operating under IEEE 802.15.4-compliant MiWi protocol from Microchip ${ }^{\circledR}$ Technology are presented. Operating tests were conducted in different hardware/firmware scenarios in order to verify the changes in node's performance depending on the adopted configuration.

KEYWORDS Energy consumption, IEEE 802.15.4 standard, MiWi protocol, sleep/idle state, wireless sensor networks.

Consideraciones acerca del consumo de energía de los nodos sensores en redes inalámbricas de área personal que utilizan el protocolo MiWi

RESUMEN La adopción de técnicas para incrementar el tiempo de operación de nodos sensores alimentados por baterías es necesaria al implementar aplicaciones prácticas de monitoreo concebidas para funcionar durante largos periodos de tiempo. La captación de energía, técnicas de convertidores dc-dc y las características de los microcontroladores modernos, como consumo extremadamente bajo, estados de reposo e inactividad, pueden contribuir al mejoramiento del desempeño de los nodos de red. Los diseñadores también pueden apoyarse en las posibilidades ofrecidas por los protocolos especiales que se utilizan en redes inalámbricas de sensores, algoritmos de enrutamiento y estrategias de adquisición y agregación de datos. Se presentan en este artículo los aspectos relacionados con el diseño e implementación de un nodo eficiente energéticamente que opere bajo el protocolo MiWi de Microchip, el cual es conforme al estándar IEEE 802.15.4. Se realizaron pruebas de operación con diferentes escenarios de hardware/firmware con el fin de verificar los cambios en el rendimiento de los nodos dependiendo de la configuración adoptada.

PALABRAS CLAVE Redes inalámbricas de sensores, estado de reposo/inactivo, consumo de energía, estándar IEEE 802.15.4, protocolo MiWi.
Considerações sobre o consumo de energia dos nós sensores das redes de área pessoal sem fio que usam o protocolo MiWi

RESUMO A adoção de técnicas para aumentar o tempo de operação de nós sensores movidos a bateria é necessária para implementar aplicações práticas de monitoramento concebidas para operar por longos períodos de tempo. A captação de energia, as técnicas de conversores dc-dc e as características dos microcontroladores modernos, como consumo extremamente baixo, estados de repouso e inatividade, podem contribuir para melhorar o desempenho dos nós da rede. Os desenhadores também podem apoiar-se nas possibilidades oferecidas pelos protocolos especiais usados nas redes de sensores sem fio, algoritmos de roteamento e estratégias de aquisição e agregação de dados. Apresentam-se neste artigo aspectos relacionados com a concepção e implementação de um nó energeticamente eficiente operando sob o protocolo MiWi da Microchip conforme o padrão IEEE 802.15.4. Foram realizados testes operacionais com diferentes cenários de hardware / firmware, com o objetivo de verificar as alterações no desempenho dos nós, dependendo da configuração adotada.

PALAVRAS-CHAVE Redes de sensores sem fios; estado de repouso/inatividade, consumo de energia, padrão IEEE 802.15.4, protocolo MiWi. 


\section{Introduction}

During the last decade, monitoring applications using Wireless Sensor Networks [WSN] based on communication standards as IEEE 802.15.4 and IEEE 802.15.1 have risen in number and variety. Applications as diverse as physiological variables monitoring, environmental measuring, and structural integrity checking are just a few examples (Fraser, Elgamal, He, \& Conte, 2009). Moreover, WSN are essential in wearable devices and Internet of Things [IoT] (Burns et al., 2010).

As a result of that, scientific literature outlines a well-defined structure for sensor nodes concerning constitutive modules. Apart from basic and traditional components, modules with microelectromechanical systems [MEMS], electronic datasheets [EDS], and energy harvesting are part of this structure (Powering microcontrollers..., 2012).

In optimal working conditions, WSN should operate unattended for long intervals by using techniques to improve energy consumption. However, in order to reach this, it is necessary to adopt design strategies from circuital and firmware approaches. As a consequence, design actions ought to be coordinated with executed code in microcontrollers, responsible to manage input/output node elements.

In this paper, I address energy efficiency topic and its relation with performance of sensor nodes, focusing in modifications related with circuital design and execution of special functions programmed in microcontrollers. My objective is to present some implementation aspects (usually not detailed) but bases of practical WSN applications and determinant to validate the assumption of operation for extended time periods.

Organization of this work is as follows: section 2 explains MiWi protocol features; in section 3, I show hardware and firmware details; implementation is showed in section 4; results discussion is presented in section 5 and section 6 briefly concludes paper.

\section{MiWi Protocol}

MiWi network protocol is property of Microchip ${ }^{\circledR}$ Technology company, and its foundation is IEEE 802.15.4 standard for Wireless Personal Area Networks [WPAN] (Microchip Technology Inc., 2010b). It proposes a light and royalty-free implementation of IEEE 802.15.4-A and it is also supported on MiMAC, a tool to handle

\section{Introducción}

Las aplicaciones de monitoreo que utilizan redes inalámbricas de sensores basadas en estándares de comunicación como IEEE 802.15.4 e IEEE 802.15.1 han aumentado en número y variedad, vertiginosamente, durante la última década. Es así como encontramos una plétora de aplicaciones que van, desde el monitoreo de variables fisiológicas y el monitoreo ambiental, hasta el monitoreo de integridad estructural, por mencionar algunas (Fraser, Elgamal, He, \& Conte, 2009). Igualmente las redes inalámbricas de sensores son parte fundamental de tecnologías como los llamados wearables (Burns et al., 2010) y del denominado Internet de las cosas [IoT].

Es así como encontramos actualmente una estructura bien establecida para los nodos sensores en cuanto a sus bloques constitutivos. Aparte de los bloques básicos tradicionales se pueden incorporar bloques con sistemas microelectromecánicos [MEMS], hojas de datos electrónicas [TEDS] y de recolección de energía [Energy Harvesting] (Powering microcontrollers..., 2012).

En la concepción óptima de las redes inalámbricas de sensores los nodos deben operar de manera inatendida por largos periodos de tiempo, basándose principalmente en un manejo eficiente del consumo de energía. No obstante, para alcanzar cifras que se acerquen al ideal teórico de rendimiento en funcionamiento, es necesario adoptar estrategias de diseño desde el punto de vista circuital y también implementar medidas a nivel del firmware. De esta forma se coordinan las acciones de diseño con el código que se ejecuta en los microcontroladores que gestionan los elementos de entrada/salida de los nodos.

En este trabajo abordaremos el tema del mejoramiento de la eficiencia energética en el desempeño de un nodo sensor a partir de tomar acciones en cuanto al diseño circuital y a la implementación de funciones especiales en el código programado en el microcontrolador. El objetivo es abordar algunos aspectos de implementación, que generalmente no son detallados, pero que son determinantes para validar el supuesto de operación sobre periodos extendidos sobre los cuales se soportan las aplicaciones prácticas de las redes inalámbricas de sensores.

La organización del trabajo es la siguiente: en la sección 2 se explican las características del protocolo MiWi; la sección 3 muestra los detalles de Hardware y de Firmware; la 4 muestra detalles de la implementación; la 5 comprende la discusión de resultados; y la 6 , presenta las conclusiones.

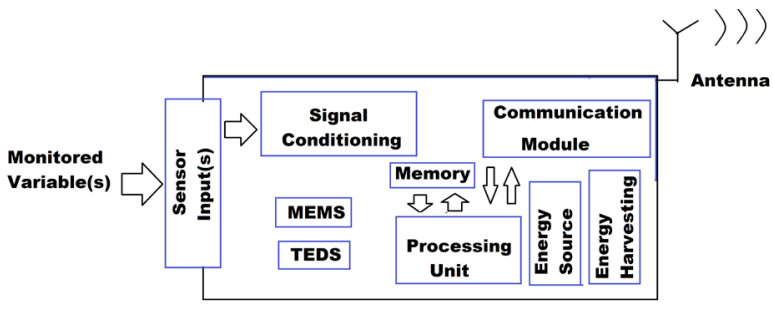

Figure 1. Block diagram of wireless sensor node / Diagrama de bloques de un nodo sensor inalámbrico 


\section{El protocolo MiWi}

El protocolo de red denominado MiWi es propietario del fabricante estadounidense Microchip y está basado en el estándar IEEE 802.15.4 para redes inalámbricas de área personal [Personal Area Networks - PAN] (Microchip Technology Inc., 2010b).

MiWi propone una implementación liviana y royalty-free, del estándar IEEE 802.15.4-A, que se apoya en una herramienta para manejar los transceptores a nivel del controlador de acceso al medio, llamada MiMAC, y una interfaz de cara al manejo de los protocolos propietarios (MiWi, MiWi P2P y MiWi PRO) llamada MiApp. MiWi está orientado a redes relativamente pequeñas, con pocos saltos: una red que utilice este protocolo puede tener un máximo de 1024 nodos.

Los nodos de red bajo MiWi pueden ser dispositivos de funciones completas [Full Function Devices - FFD] o dispositivos de funciones reducidas [Reduced Function Devices - RFD]; jerárquicamente el nodo principal de la red de área personal, denominado $P A \mathcal{N}$ Coordinator, debe ser siempre un FFD. El PAN Coordinator siempre es único, incluso en topologías como malla (mesh) o cluster tree, en las que hay coordinadores secundarios en las derivaciones de red, denominados simplemente Coordinadores. Tanto el Coordinador de la PAN como los demás coordinadores siempre son FFD. Cada coordinador puede tener máximo 127 nodos a su cargo o hïos, con un máximo de 8 coordinadores en una red. Los paquetes pueden viajar un máximo de 4 saltos en la red y 2 desde el coordinador de la PAN (Microchip Technology Inc., 2010b).

\section{A. MiWi P2P}

Dado que nuestro foco de atención en el tipo de aplicaciones que requieren pocos nodos (menos de 127) y con comunicación para rangos de distancias menores a 120 metros, nos centraremos en el protocolo MiWi P2P (Microchip Technology Inc., 2010a). MiWi P2P modifica la capa de control de acceso al medio [MAC] de la especificación IEEE 802.15.4, simplificando así el proceso de handshaking o establecimiento de la comunicación. Este protocolo soporta las topologías P2P y estrella. No posee mecanismo de enrutamiento, de modo que el cubrimiento de la comunicación inalámbrica se define mediante el rango del radio. No es multihop (multisalto). El protocolo MiWi P2P solo soporta redes sin beacon (baliza).

\section{B. Topología estrella}

Desde la perspectiva del rol de un dispositivo, la topología tiene un Coordinador de la PAN que inicia la comunicación

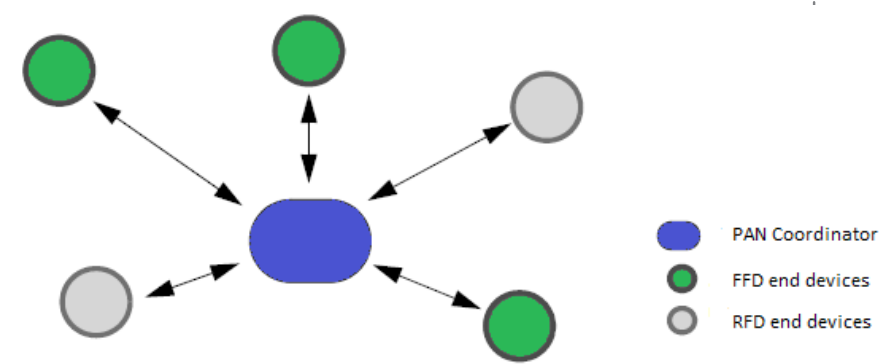

Figure 2. Star topology under MiWi P2P (Microchip Technology Inc., 201'Oa) / Topología estrella bajo el protocolo MiWi P2P (Microchip Technology Inc., 2010a) transceivers at Media Access Control [MAC] level. Other feature is related with an interface featuring Microchip ${ }^{\circledR}$ proprietary protocols (MiWi, MiWi P2P, and MiWi PRO) called MiApp. MiWi is oriented to relatively small networks with few hops (i.e. networks using this protocol can reach up to 1024 nodes).

Network nodes under MiWi can be Full Function Devices [FFD] or Reduced Function Devices [RFD]; hierarchically, main node in Personal Area Network [PAN], called PAN coordinator, must always be an FFD. PAN coordinator is always unique, even in mesh or cluster tree topologies, where secondary coordinators in network derivations might exist (called only coordinators). These coordinators are also FFD nodes and each one supports up to 127 nodes (children nodes), with a maximum number of 8 coordinators in the network. Packets are able to travel until 4 hops in the network and 2 hops from PAN coordinator (Microchip Technology Inc., 2010b).

\section{A. MiWi P2P}

Since my focus is low-node applications (under 127) and communication ranges smaller than 120 meters, I concentrated this work on the MiWi protocol (Microchip Technology Inc., 2010a). MiWi P2P modifies MAC layer of IEEE 802.15.4, simplifying handshaking (communication establishment) process and it supports $\mathrm{P} 2 \mathrm{P}$ and star network topologies. It does not have a routing mechanism; hence, the radio range defines wireless communication coverage. In addition, MiWi P2P is not multi-hop and it supports only beaconless networks.

\section{B. Star topology}

In relation to role perspective in devices, the topology has a PAN coordinator, which starts communication and accepts remote connections from other devices. Several end devices join this communication and they can only establish connections with the PAN coordinator, not among them. As mentioned previously, the PAN coordinator is a FFD device; nevertheless, end devices can be FDD with their radio constantly on, or they can assume RFD roles with their radio off when the idle state is reached. Regardless of their functional style, end devices can only communicate with the coordinator, whilst he coordinator can communicate with every node presented in the network (Microchip Technology Inc., 2010a).

\section{Network addressing}

Network addresses are assigned having as a fixed one value 11-22-33-44-55-66-77-01 for the coordi- 




Figure 3. Addressing fields in packet format over MiWi P2P protocol (Microchip Technology Inc., 2010a) / Campos de direccionamiento en el formato de los paquetes del protocolo MiWi P2P (Microchip Technology Inc., 2010a)

nator. The assignment for sensor nodes starts from 11 22-33-44-55-66-77-02 and so forth. Network identification address [PAN ID] is, by default, 1234.

MiWi P2P protocol only supports one-hope communications; thus, message transmission is carried out using Extended Unique Identifier [EUI] address (i.e. the long address). Broad diffusion messages (broadcast) use short addressing only. For Microchip ${ }^{\circledR}$ Technology transceivers, the size of the unique address is between 2 and 8 bytes, depending on application necessities. The message format in MiWi P2P protocol derives from the one specified in IEEE 802.15.4 standard. Figure 3 illustrates package format and its fields (Microchip Technology Inc., 2010a).

\section{Firmware and hardware relative aspects}

There are some research projects related to performance evaluation of nodes in MiWi networks; so, this work is intended to focus in some aspects where these projects do not emphasize. In one of these proposals, the authors analyze a network conformed of 7 nodes and measure current in transmission, reception, and idle states, obtaining values between 60 and $95 \mathrm{~mA}$. Adoption of hardware/firmware actions to reduce energy consumption is not reported (Portillo-Rodríguez, Alcaide-Barragán, Sandoval-González, Vilchis-Gonzalez, \& Ávila-Vilchis, 2011). In my research, I also found a paper that analyzes case study of nodes powered by solar panels where, at firmware level, they are programmed to access the sleep mode to reduce energy consumption. Nevertheless, authors show neither the node's current consumption nor extended operation tests; the study is focused on performance evaluation of energy acquisition in a specific time (6AM-6PM) (Gargiulo et al., 2010). In addition, I analyzed the work conducted by Pannila, Tuominen, and Edirisinghe (2011); they propose combined use of Radio-frequency Identification [RFID] tags with MiWi protocol to get data from gas sensors. In this work, the y acepta conexiones de otros dispositivos. Se tienen varios dispositivos terminales (end devices) que se unen a la comunicación. Los dispositivos terminales pueden establecer conexiones solo con el coordinador de la PAN, no entre ellos. En cuanto al tipo de funcionalidad, el coordinador de la PAN es un dispositivo FFD. Un dispositivo terminal puede ser un FFD con su radio encendido todo el tiempo o un dispositivo de funciones reducidas RFD con su radio apagado cuando está inactivo -idle-. Sin importar su tipo funcional, los dispositivos terminales solo se pueden comunicar con el coordinador, mientras que el coordinador si puede comunicarse con todos (Microchip Technology Inc., 2010a).

\section{Direccionamiento de la red}

Las direcciones de red se asignan teniendo como fija para el coordinador de la red la dirección 11-22-33-44-55-66-7701. Para los nodos sensores se asignan a partir del elemento 11-22-33-44-55-66-77-02 y así sucesivamente. La dirección de identificación de la red (PAN ID) será por defecto 1234.

El protocolo MiWi P2P solo soporta comunicación de un salto, de allí que solo se transmitan mensajes con la dirección EUI o larga. El direccionamiento corto se utiliza solo cuando se transmite un mensaje de difusión amplia [broadcast]. Para los transceptores de microchip, la longitud de la dirección única puede estar entre 2 y 8 bytes, dependiendo de las necesidades de la aplicación. El formato del mensaje en el protocolo MiWi $\mathrm{P} 2 \mathrm{P}$ se deriva del formato de mensaje de la especificación IEEE 802.15.4. La Figura 3 ilustra el formato de paquete y sus campos (Microchip Technology Inc., 2010a).

\section{Aspectos relativos al firmware y al hard- ware}

Existen en la literatura algunos trabajos relacionados con la valoración del desempeño de los nodos en redes MiWi, este trabajo pretende ahondar es algunos aspectos de los que no se encontraron mayores detalles. En uno de estos trabajos se analiza una red de siete nodos y se mide la corriente en periodos de transmisión, recepción y espera, oscilando este valor entre unos 60 y $95 \mathrm{~mA}$. En este trabajo no se reporta la adopción de medidas de hardware o firmware para reducir el consumo de energía (Portillo-Rodríguez, Alcaide-Barragán, Sandoval-González, Vilchis-Gonzalez, \& Ávila-Vilchis, 2011). También se ha encontrado un trabajo que analiza el caso de un nodo alimentado con un panel solar fotovoltaico, el cual, a nivel de firmware, accede al modo sleep para reducir el consumo de energía; no obstante, no se indican detalles de consumo de corriente del nodo ni de pruebas de operación extendida, 
sino que el análisis se centra en el rendimiento del medio de recolección de energía en el periodo 6AM-6PM (Gargiulo et al., 2010). Adicionalmente se analizó un artículo que propone el uso conjunto de etiquetas de identificación por radiofrecuencia [RFID] y el protocolo MiWi para recoger los datos entregados por sensores de gas. En este trabajo se reconocen los beneficios que se pueden derivar del consumo reducido de los nodos MiWi en modo sleep, sin embargo, no se reporta ningún resultado experimental o verificación al respecto (Pannila, Tuominen, \& Edirisinghe, 2011).

\section{A. Consideraciones relativas al hardware}

Denominamos hardware al conjunto de dispositivos físicos que hacen parte del circuito de los nodos de una red inalámbrica de sensores. En particular nos centraremos en el análisis de los elementos circuitales de un nodo sensor y en la manera cómo influyen sobre el desempeño de este, desde el punto de vista del consumo de energía. A continuación presentamos algunas consideraciones relativas a las modalidades de diseño que tienen que ver con el hardware de los nodos.

- Conversores DG-DG. Circuitalmente es común encontrar enfoques de diseño centrados en el conversor DC-DC que provee la alimentación de voltaje normalizado para el nodo, como por ejemplo $3.3 \mathrm{~V}$ a partir de un batería. Las notas de aplicación de diferentes fabricantes proveen indicaciones acerca de conversores tipo step-up o step-down conmutados que se apoyan en circuitos integrados de uso específico e inductores.

- Energy harvesting. Los dispositivos o medios de recolección de energía (fotovoltaicos, térmicos, piezoeléctricos, vibracionales, etc.) son una opción de hardware para mejorar la autonomía energética de los nodos. Un aspecto a tener en cuenta es que el tipo de aplicación determina, en gran medida, la opción u opciones de energy harvesting aplicables en cada situación. En aplicaciones en las que los nodos sensores están en movimiento, ya sea porque los portan personas o porque están implementados sobre vehículos o similares, los medios de energy harvesting, piezoeléctricos y basados vibraciones son viables. Otro aspecto determinante es el entorno de la aplicación, es decir, si esta se realiza en interiores (indoor) o en exteriores (outdoor), y si se despliegan los nodos en entornos urbanos, rurales o industriales, porque está ligado a la posibilidad de recurrir a opciones como la energía solar fotovoltaica para complementar el suministro de energía de los nodos.

La adopción de elementos de recolección de energía, como parte del hardware de los nodos, resulta en un incremento en el tamaño y peso de los nodos, aparte de afectar aspectos de precio, flexibilidad y mantenimiento, que deben ser analizados concienzudamente, según el ámbito de cada aplicación. Adicionalmente, un factor a considerar es el estado actual de madurez de los diferentes dispositivos y tecnologías de recolección de energía, en ese sentido, es evidente que actualmente la opción solar fotovoltaica es una de las tecnologías más afianzadas, authors recognize benefits derived from reduced consumption of MiWi nodes in sleep mode; notwithstanding, no experimental results or verifications are shown.

\section{A. Hardware relative considerations}

As readers might infer, we usually call hardware to the set of physical devices, in this case, conforming nodes' circuits in a wireless sensor network. Particularly in this work, my focal point is the analysis of circuital elements of sensor nodes and the manner they have influence over general energy consumption performance. In the following paragraphs, I present some considerations relative to design approaches associated with hardware in nodes.

- DG-DC converters. Relative to circuitry, it is common to find design methods centered on DG-DG converters, which deliver normalized voltage for nodes (e.g. 3.3V provided by Li-ion batteries). Application notes from several manufacturers give general information about step-up and step-down switched converters, which are supported in specific-use integrated circuits and inductors.

- Energy harvesting. Energy harvesting devices (photovoltaic, thermal, piezoelectric, vibrational, etc.) are a good hardware option to improve energy autonomy in nodes. In general, application type mainly determines energy harvesting options applicable in varied situations. For instance, in certain applications where sensor nodes are moving (either carried by people or within vehicles), piezoelectric and vibrational energy harvesting options are the most suitable. Another important aspect is related with location of sensors, i.e. indoors, outdoors, urban, rural, or industrial environments. Depending on this location, photovoltaic energy sources might be used to complement main supply in sensor nodes.

Adoption of energy harvesting elements results on rises in size and weight of nodes; besides, this approach affects price, flexibility, and maintenance. Furthermore, another relevant factor is maturity in devices and technologies used in energy harvesting. Therefore, it is evident that photovoltaic option is one of the most consolidated technologies comparing with other developing elements.

- Components selection. Regarding design in nodes' circuitry, continuous operation over extended time intervals is desirable; further, it is important 
to compare specifications given by technical datasheets. As an example, microcontrollers present several references with low power consumption and extra-reduced size, making them optimal for battery-operated applications. In addition to that, batteries present various features that are particularly useful for certain applications. Lithium-ion [Li-ion] and lithium polymer [LiPo] batteries are very popular for their performance, although they are more expensive than other types like nickel-metal hydride $[\mathrm{NiMH}]$ or popular alkaline batteries.

- Other strategies. Adoption of techniques like switching in sensors energy supply (which might lead to high prices and, consequently, affect budget), could significantly contribute to energy efficiency, resulting in optimal performance over long periods. The cost of maintaining switching circuits must be considered because switching is not electronic and, commonly, it is expensive from an energy point of view.

\section{B. Firmware relative considerations}

Code executed by a microcontroller that controls actions in nodes is also a key aspect. Throughout it, functions and special resources in a microcontroller can be accessed and, in terms of MiWi protocol, this code provides access to special features over MiApp interface and MiMAC peculiarities (Microchip Technology Inc., 2009).

Eight bit microcontrollers like PIC18F4620 (Microchip Technology Inc., 2004) manufactured by Microchip ${ }^{\circledR}$ Technology, allow operation in low-power states using sleep instruction. With respect to energy consumption, Run, Idle and Sleep operation states are defined, distinguishing among them by CPU resource management and peripherals. TABLE 1 illustrates the resource management over these three states.

The OSCGON register presented in PIC18F4620 permits you to choose between Idle and Sleep modes (Microchip Technology Inc., 2004). There are several ways to exit these modes:

- enabling one or more interruption sources;

- watch dog timer overflow; and

- exit forcing restart (reset).

MiApp. It defines programming interfaces between application layer and wireless proprietary protocols of Microchip ${ }^{\circledR}$ Technology (Microchip Technology Inc., 2009). There are some configuration parameters and a en comparación con otras tecnologías emergentes o en camino de consolidación.

- Selección de los componentes. Cuando se trata de diseñar los circuitos de nodos que se pretende que funcionen de manera continua sobre periodos extendidos de tiempo, es importante tomarse un tiempo para comparar las especificaciones que detallan, en las hojas de datos, los diferentes fabricantes de componentes electrónicos. Los microcontroladores, por ejemplo, presentan referencias de consumo reducido y extra-reducido que se acomodan mejor a las aplicaciones alimentadas por baterías. Igualmente las baterías presentan características que las hacen más adecuadas para cierto tipo de aplicaciones. Las baterías de Litio-Ion y Litio-Polímero son muy populares por su rendimiento, pero continúan siendo más costosas que otros tipos, como las de Ni-MH o las también populares baterías alcalinas.

- Otras estrategias. La adopción de técnicas, como la conmutación del suministro de energía de elementos como los sensores, que pueden tener un consumo significativo dentro del presupuesto global del nodo sensor, puede contribuir de manera importante en la eficiencia energética de cara a un funcionamiento sobre periodos extendidos de tiempo. Debe valorarse el costo energético de mantener operando los circuitos de conmutación, puesto que la conmutación que no es electrónica suele ser costosa desde el punto de vista energético.

\section{B. Consideraciones relativas al firmware}

El código ejecutado por el microcontrolador que gobierna las acciones del nodo también es un aspecto clave. A través de él, se puede acceder a funciones y recursos especiales del mismo microcontrolador y, en el caso del protocolo MiWi, a características especiales a través de la interfaz MiApp [Microchip Wireless Application Programming Interface] y de las funciones de MiMAC [Microchip Wireless Media Access Controller] (Microchip Technology Inc., 2009).

Los microcontroladores de 8 bits, como el PIC18F4620 (Microchip Technology Inc., 2004) del fabricante Microchip permiten acceder a modos de consumo reducido mediante la instrucción sleep. En cuanto a consumo de energía, se denominan los estados de operación del microcontrolador como Run, Idle y Sleep, diferenciándose por la gestión que hace de los recursos de la CPU y de los periféricos, en cada caso. La TABLA 1 ilustra la gestión de los recursos en los tres modos descritos.

Table 1. Microcontroller operating modes based on energy consumption Modos de funcionamiento del microcontrolador según el consumo de energía

\begin{tabular}{llc}
\hline $\begin{array}{l}\text { Microcontroller } \\
\text { state / Estado del mi- } \\
\text { cro-controlador }\end{array}$ & \multicolumn{2}{c}{ Resources management / Gestión } \\
\cline { 2 - 3 } & \multicolumn{2}{c}{ CPU los recursos } \\
\hline Run & ON & $\begin{array}{c}\text { Peripherals } \\
\text { Perifericos }\end{array}$ \\
\hline Idle & OFF & ON \\
\hline Sleep & OFF & OFF \\
\hline \hline
\end{tabular}


El registro OSCGON del PIC18F4620 permite escoger entre los modos Idle y Sleep (Microchip Technology Inc., 2004). Para salir de estos modos existen diferentes mecanismos:

- activación de una de las fuentes de interrupción habilitadas;

- desborde del perro guardián (Watch Dog Timer); y

- salida por reinicio (reset).

MiApp. Define las interfaces de programación entre la capa de aplicación y los protocolos inalámbricos propietarios de Microchip (Microchip Technology Inc., 2009). En la implementación de MiApp se encuentran parámetros de configuración y un conjunto de funciones disponibles en el proyecto de MPLAB que se compilan al programar los nodos de las redes MiWi. Mediante los parámetros de configuración se definen aspectos como el transceptor a utilizar y así, indirectamente, la banda de operación, el protocolo (MiWi, MiWiP2P, etc.), el tamaño del buffer, el escaneo del canal menos ruidoso para transmitir y el rol del nodo (coordinador o dispositivo terminal), entre otros. Las funciones MiApp permiten, entre otros, acceder a:

- el canal de operación (en la banda de 2.4GHz del 11 al 26);

- buscar, iniciar y remover una conexión;

- enviar y recibir mensajes;

- manejar el consumo de energía del transceptor; y

- channel hopping -llamado frequency agility en MiWi-.

La llamada a la función Mi_AppTransceiverPowerState se define para poder poner el transceptor en modo sleep periódicamente:

BYTE MiApp_TransceiverPowerState(BYTE Mode);

El único parámetro de la función es el modo de operación. Los modos de operación predefinidos (Microchip Technology Inc., 2009) son:

- POWER_STATE_SLEEP: coloca el transceptor en modo Sleep;

- POWER_STATE_WAKEUP: despierta el transceptor sin enviar ninguna petición de datos; y

- POWER_STATE_WAKEUP_DR: despierta el transceptor y luego envía una petición de datos a su dispositivo asociado principal para preguntar por datos entrantes.

MiMAC. La capa de controlador de acceso al medio provee los canales de acceso básicos, el direccionamiento y las funcionalidades de transmisión/recepción en la parte superior de la capa PHY en la que se maneja los datos rasos (raw data) (Microchip Technology, 2009). A través de las definiciones y funciones de MiMAC se puede acceder, a un nivel más bajo, a características como las siguientes:

- cifrado de los mensajes;

- potencia del transceptor -en algunos casos con ciertas referencias de radios MiWi-;

- canal de operación;

- evaluación de ruido sobre el canal;

- estado de consumo de energía del transceptor set of functions available in the created MPLAB project, which are compiled by programming nodes in MiWi networks. By means of configuration parameters, system defines aspects like transceiver to use; subsequently, it also defines operation band, protocol (MiWi, MiWiP2P, etc.), buffer size, less noisy channel by scanning, and node role (coordinator or end device), among other points. MyApp functions let access to the following:

- operation cannel (in 2.4GHz band, from cannel 11 to channel 26);

- search, start, and remove connections;

- send and receive messages;

- manage transceiver energy consumption; and

- channel hoping (called frequency agility in MiWi).

Invoking the "Mi_AppTransceiverPowerState" function means to put the transceiver into Sleep mode periodically:

\section{BYTE MiApp_TransceiverPowerState(BYTE Mode);}

The only parameter in the previous function is the operation mode. Predefined operation modes (Microchip Technology Inc., 2009) are:

- POWER_STATE_SLEEP: puts transceiver in Sleep mode;

- POWER_STATE_WAKEUP: wakes up transceiver without any data request; and

- POWER_STATE_WAKEUP_DR: not only wakes up transceiver, but also sends data request to its main associated device to ask for incoming data.

MiMAC. Media access control layer supplies basic access channels, addressing, and reception/transmission functionalities on top of physical layer (where raw data is handled) (Microchip Technology, 2009). Using definitions and functions in MiMAC, user can access at a lower level to the following characteristics:

- message encryption;

- transceiver power;

- operation channel;

- noise evaluation over channel; and

- energy consumption state of transceiver

Calling to the "MiMAC_PowerState" function specifies the desired energy state for the transceiver: 
PowerState is the only input parameter and it has one of the two following states (Microchip Technology Inc., 2009):

- DEEP_SLEEP: selects sleep state for transceiver, using lowest energy as possible; and

- OPERATE: transceiver normal operation state.

Figure 4 shows, in a more simplistic way, the integration between MiApp interface and MiMAC layer in the process of programming an application over MiWi protocol.

\section{Methodology}

The sensor node used to verify energy consumption conditions under several configurations, both at hardware and firmware levels, is based on an 8-bit microcontroller, a MiWi transceiver, and a 6 degrees of freedom inertial measurement unit [IMU]. Specifically, I used a PIC18F4620 microcontroller (Microchip Technology Inc., 2004), MRF24J40MA transceiver (Microchip Technology Inc., 2008), and an IMU consisting of accelerometer and gyroscope over X, Y, and Z axes. Complements to sensor nodes are an $8.4 \mathrm{~V}$ rechargeable battery and a $3.3 \mathrm{~V}$ regulated power supply. TABLE 2 shows the technical specifications of these devices.

TABLE 3 presents real measurements of current supplied to node circuitry in a stand-by situation. When

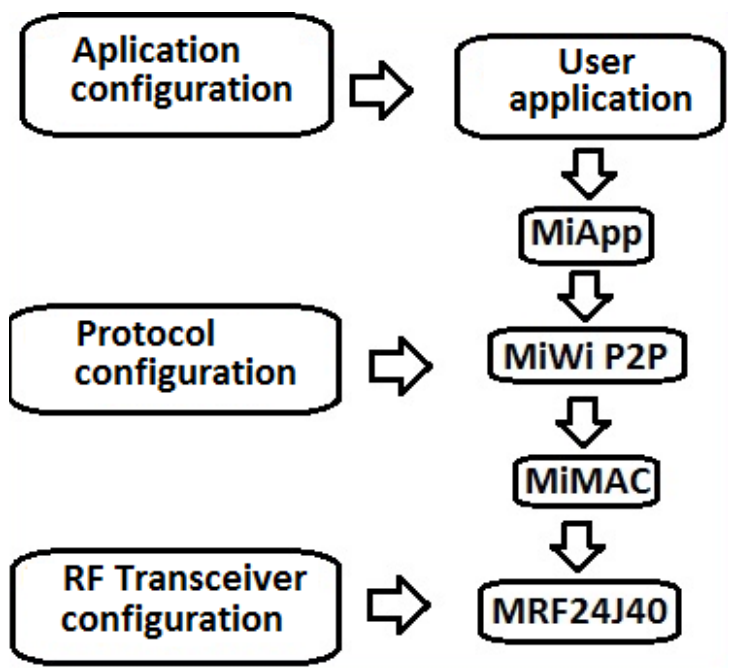

Figure 4. Integration diagram in development of applications with MiMAC and MiApp / Diagrama de integración en el desarrollo de aplicaciones con las herramientas MiMAC y MiApp del stack MiWi de Microchip

La llamada a función MiMAC_PowerState permite indicar el estado deseado de energía para el transistor.

BOOL MiMAC_PowerState(BYTE PowerState)

El único parámetro de entrada PowerState puede tomar una de dos posibilidades (Microchip Technology Inc., 2009):

- DEEP_SLEEP: selecciona el estado Sleep para el transceptor con el consumo de energía más bajo; y

- OPERATE: operación en estado pleno del transceptor.

La FIGURA 4 muestra, de manera simplificada, cómo se integran la interfaz MiApp y MiMAC en el proceso de programación de una aplicación bajo el protocolo MiWi.

Table 2. Devices and their technical specifications / Dispositivos y sus especificaciones técnicas

\begin{tabular}{|c|c|}
\hline Device / Dispositivo & Main technical specs / Características técnicas principales \\
\hline \multirow{5}{*}{$\begin{array}{l}\text { PIC18F4620 microcontroller / } \\
\text { Microcontrolador PIC18F4620 }\end{array}$} & Operation voltage: $3.3 \mathrm{~V} /$ Voltaje de operación: $3.3 \mathrm{~V}$ \\
\hline & Program memory: $64 \mathrm{~KB} /$ Memoria de programa: $64 \mathrm{~K}$ \\
\hline & Data memory: $1 \mathrm{~KB}$ of EEPROM and 3.875KB of SRAM / Memoria de datos: $1 \mathrm{~K}$ de EEPROM y 3968 bytes de SRAM \\
\hline & Minimal current in Sleep mode: 100nA / Corriente mínima en modo sleep: $100 \mathrm{nA}$ \\
\hline & A/D conversion channels: 10 channels, each of 10 bits / Canales de conversión A/D: 10 canales de 10 bits. \\
\hline \multirow{6}{*}{$\begin{array}{l}\text { MRF24J40MA transceiver / } \\
\text { Transceptor MRF24f40MA }\end{array}$} & Operation voltage: $3.3 \mathrm{~V} /$ Voltaje de operación: $3.3 \mathrm{~V}$ \\
\hline & Interface: four line SPI / Interfaz: SPI de 4 lineas \\
\hline & Current during transmission: $23 \mathrm{~mA}$ / Corriente durante transmisión: $23 \mathrm{~mA}$ \\
\hline & Current during reception: $19 \mathrm{~mA} /$ Corriente durante recepción: $19 \mathrm{~mA}$ \\
\hline & Current in Sleep mode: $2 \mu \mathrm{A}$ (nominal) / Corriente en modo sleep: $2 \mu \mathrm{A}$ típico \\
\hline & Operation band: $2.4 \mathrm{GHz}$ (ISM) / Banda de operación (ISM): $2.4 \mathrm{GHz}$ \\
\hline \multirow{4}{*}{$\begin{array}{l}\text { IMU sensor / Sensor IMU de } 6 \text { grados } \\
\text { de libertad }\end{array}$} & Operation voltage: $3.3 \mathrm{~V} /$ Voltaje de operación: $3.3 \mathrm{~V}$ \\
\hline & 2-axes gyroscope: LPR530A / Giróscopo de 2 ejes: LPR530A \\
\hline & 1-axis gyroscope: LY530ALH / Giróscopo de 1 eje: LY530ALH \\
\hline & 3-axes accelerometer: ADXL335 / Acelerómetro de 3 ejes: ADXL335 \\
\hline \multirow[t]{3}{*}{ Battery / Batería } & Type: rechargeable / Tipo: recargable \\
\hline & Type: NiMH / Compuesto: NiMH \\
\hline & Voltage: $8.4 \mathrm{~V} /$ Voltaje: $8.4 \mathrm{~V}$ \\
\hline \multirow{4}{*}{$\begin{array}{l}\text { LD1 } 117 \text { voltage regulator (ST, } \\
\text { 2013) / Regulador de voltaje LD1117 } \\
\text { (ST, 2013) }\end{array}$} & Output voltage: $3.3 \mathrm{~V} /$ voltaje de salida: $3.3 \mathrm{~V}$ \\
\hline & Maximum output current: $800 \mathrm{~mA}$ (nominal) / Corriente de salida máxima: $800 \mathrm{~mA}$ (típico) \\
\hline & Maximum input voltage: $15 \mathrm{~V} /$ Voltaje máximo de entrada: $15 \mathrm{~V}$ \\
\hline & Dropout voltage: from 1 to $1.2 \mathrm{~V} /$ Dropout voltaje: de 1 a $1.2 \mathrm{~V}$ \\
\hline
\end{tabular}




\section{Metodología}

El nodo sensor que se utilizó para verificar las condiciones de consumo de energía bajo diferentes configuraciones, tanto a nivel de hardware, como de firmware, se basa en un microcontrolador de 8 bits, un transceptor MiWi y una unidad de medición inercial de 6 grados de libertad. Específicamente, se utilizó un microcontrolador PIC18F4620 (Microchip Technology Inc., 2004), un transceptor MRF24J40MA (Microchip Technology Inc., 2008) y una IMU que agrupa un acelerómetro y un giróscopo en los ejes $\mathrm{X}, \mathrm{Y}$, Z. Complementan el nodo sensor: una batería recargable de 8.4 voltios y un regulador de voltaje de 3.3V. La TABLA 2 resume las especificaciones técnicas principales de dichos dispositivos.

La TABLA 3 muestra lo que indican las mediciones de la corriente suministrada al conjunto circuital del nodo en una situación standby. Cuando se da la transmisión de un paquete con esta configuración, el consumo total se acerca a $50 \mathrm{~mA}$, aunque alcanza, en algunos momentos, un valor de $47.2 \mathrm{~mA}$.

Claramente, el nodo presenta un consumo elevado en dos elementos que pueden ser controlados, de alguna forma, por medio de hardware o firmware, el radio transceptor y el elemento sensor IMU. En primer lugar se decidió conmutar la conexión del sensor para que el consumo de corriente no sea permanente, sino que se presente solo cuando se realice la conversión análoga/digital en el microcontrolador. Para conmutar el sensor se probó inicialmente con un transistor bipolar (BJT) de uso común, que interrumpía la conexión a uno de los extremos del suministro de voltaje cuando la toma de datos de medición estaba inactiva. Este método mostró no ser eficiente puesto que las mediciones entregadas por el sensor se degradaban, dado que la conmutación no era total y, por ende, el voltaje de alimentación del sensor no era el nominal. Para tratar de resolver esto se probó, tanto la conmutación del polo positivo, como la del polo de tierra, así como la utilización de un par Darlington y de un interruptor bilateral, sin obtener resultados favorables. La conmutación se comandó desde un pin del microcontrolador que sincronizaba la conmutación con los momentos de lectura de los canales de conversión A/D de entrada.

Considerando los resultados poco satisfactorios obtenidos, tanto con lo BJT, como con los interruptores bilaterales, se eligió un transistor de efecto de campo para realizar la conmutación. El FET elegido, un transistor de efecto de campo de canal $\mathrm{N}$ de referencia 2N7000 (Fairchild Semiconductor, 1997), presentó un desempeño favorable. Para verificar el correcto funcionamiento del circuito con la conmutación del sensor incorporada, se procedió a dejar el nodo sensor en funcionamiento durante varias horas, en diferentes sesiones de trabajo, obteniendo resultados satisfactorios al elegir un temporizado suficiente para mantener la conmutación activa.

La TABLA 4 resume los resultados en cuanto a consumo de corriente del nodo luego de la conmutación del sensor IMU.

Con el fin de optimizar el consumo de energía sobre los periodos que hemos denominado modo continuo, que corresponden a los periodos en que permanece principalmente el nodo sensor, se optó por verificar las prestaciones de consumo reducido de energía que ofrece el microcontrolador.
Table 3. Global current consumption of devices in sensor node / Consumo global de corriente de los dispositivos que conforman el nodo sensor

\begin{tabular}{lr}
\hline Device / Dispositivo & \multicolumn{1}{c}{$\begin{array}{l}\text { Current consump- } \\
\text { tion / Consumo de corriente }\end{array}$} \\
\hline \hline $\begin{array}{l}\text { PIC18F4620 microcontroller / } \\
\text { Microcontrolador PIC18F4620 }\end{array}$ & $4.4 \mathrm{~mA}$ \\
\hline $\begin{array}{l}\text { MRF24J40MA transceiver / Transcep- } \\
\text { tor MRF24740MA }\end{array}$ & $15 \mathrm{~mA}$ \\
\hline IMU sensor / Sensor IMU & $16.4 \mathrm{~mA}$ \\
\hline Voltage regulator / Regulador de voltaje & $6 \mathrm{~mA}$ \\
\hline Total & $41.8 \mathrm{~mA}$ \\
\hline
\end{tabular}

packets are transmitted over this configuration, total consumption is, on average, $40 \mathrm{~mA}$; although in some cases, system reaches peaks up to $47.2 \mathrm{~mA}$.

Clearly, the studied node presents high energy consumption in two elements, somehow controlled by the hardware or firmware: radio transceiver and IMU sensor. Firstly, I decided to switch the sensor connection in order to avoid continuous current consumption; only when analog/digital conversion takes place in microcontroller, is the sensor connection on. To achieve this switching, I employed as first option a Bipolar Junction Transistor [BJT], which interrupts connection to one of two external voltage supplying sources when data transferring was inactive. This method showed inefficiency, since delivered measurements from sensor presented degradations given partial switching; in consequence, supplying voltage was not nominal. To solve this, I tried both positive pole and ground pole switching, together with a Darlington pair and bilateral interrupters, without obtaining favorable results. Switching was commanded from microcontroller pin that synchronizes this task with lecture moments of analog/digital conversion channels.

Considering unsatisfactory results with $\mathrm{BJT}$ and bilateral interrupters, I decided to use a Field Effect Transistor [FET] to implement switching process. I used N-channel FET with reference 2N7000 (Fairchild Semiconductor, 1997), and it led to successful results. In order to verify circuit appropriate operation, procedure consisted in allowing uninterrupted operation in sensor node for several hours; this produced satisfactory results since chose time was enough to maintain switching active. TABLE 4 resumes results related with node current consumption after switching in IMU sensor.

In order to optimize energy consumption over specific time periods (named continuous mode), which correspond to intervals where sensor node is active, I verified 
Table 4. Current consumption in transceiver with sleep mode / Consumo de corriente con modo sleep en el transceptor

\begin{tabular}{lcc}
\hline Sensor node (conditions) / Nodo sensor & \multicolumn{2}{c}{ Current consumption (mA) / Consumo de corriente (mA) } \\
\cline { 2 - 3 } & Continuous mode / Modo continuo & Transmission / Transmisión \\
\hline $\begin{array}{l}\text { Sensor switching process and transceiver in } \\
\text { sleep mode after transmission / Conmutación del } \\
\text { sensory Radio Transceptor entra en modo sleep durante luego de } \\
\text { transmitir }\end{array}$ & $23.8 \mathrm{~mA}^{*}$ & $29.4 \mathrm{~mA}$ \\
\hline * During short periods, system presented values of 15,19 , and 20mA. / * Durante periodos muy cortos se obtienen valores de 15, 19y 20mA
\end{tabular}

Table 5. Current consumption with sleep mode in transceiver and microcontroller, besides of sensor switching / Consumo de corriente con modos sleep en el transceptor y el microcontrolador, y conmutación del sensor

\begin{tabular}{lcc}
\hline \hline \multicolumn{1}{c}{$\begin{array}{c}\text { Sensor node (conditions) / Nodo sensor } \\
\text { (Condiciones) }\end{array}$} & Current consumption / Consumo de corriente \\
\cline { 2 - 3 } & Continuous mode / Modo continuo & $\begin{array}{c}\text { Transmission / Trans- } \\
\text { misión }\end{array}$ \\
\hline $\begin{array}{l}\text { Sensor switching process and transceiver in } \\
\text { sleep mode after transmission; besides, micro- } \\
\text { controller also in sleep mode. / Commutación del sensor } \\
\text { yel radio transceptor entra en modo sleep durante luego de transmitir, } \\
\text { igualmente el microcontrolador también ingresa al estado sleep. }\end{array}$ & $6.1 \mathrm{~mA}$ & \\
\hline
\end{tabular}

the reduced consumption data that the microcontroller offers.

Considering that the main objective of this proposal is to obtain the lowest consumption possible, sleep mode activation is programmed via invocation from code execution with the same name (sleep). The IDLEN bit from the OSCCON register permits enabling of input in sleep mode, whilst exiting from this mode is possible in several ways. In this case, exit of this state is activated through overflowing method called Watchdog Timer [WDT]. As a result of that, postscaler of WDT was configured in several relations, until obtaining an adequate periodicity in transmission. CONFIG2H register and its WDTPS3 and WDTPS0 bits were modified to check different relations in postscaler, since they start in 1:32768, continue to $1: 16384$ and so forth.

The best conditions related with node current consumption are achieving programming in both the microcontroller and transceiver sleep mode condition, together with "Mi_AppTransceiverPowerState()" function (Microchip Technology Inc., 2009). The IMU sensor only turns on to gather data; after that, it is powered off by the FET. Detailed results of current consumption are shown in TABle 5; in addition, Figure 5 shows a photograph of utilized prototype working over conditions described in previous two tables.

Comparing data in Table 4 and Table 5, results suggests a noticeable reduction in current consumption from the sensor node in continuous mode. This reduction is mainly because of the sleep state of both
Puesto que se desea obtener el menor consumo posible, se programa en el microcontrolador la habilitación del modo sleep cuando este sea invocado desde la ejecución del código con la función del mismo nombre. El bit IDLEN del registro OSCCON permite habilitar la entrada en modo sleep, mientras que la salida de dicho modo se puede lograr por diferentes vías, en este caso, se activa la salida de este estado mediante el mecanismo de desbordamiento del denominado Perro Guardián (Watchdog Timer-WDT); se configuró el postscaler del WDT en diferentes relaciones, hasta tener una periodicidad en los envíos que se consideró adecuada. El registro CONFIG2H y sus bits WDTPS3 a WDTPS0 fueron modificados para revisar las diferentes relaciones del postcaler que comienzan 1:32768, luego pasan a 1:16384, y así sucesivamente.

Programando en el microcontrolador la condición de modo sleep y habilitando la misma condición en el radio transceptor, con la función Mi_AppTransceiverPowerState() (Microchip Technology Inc., 2009), se lograron las mejores condiciones, en cuanto a consumo de corriente del nodo. El sensor IMU solo se conecta para adquirir sus datos y se desconecta luego mediante el transistor de efecto de campo.
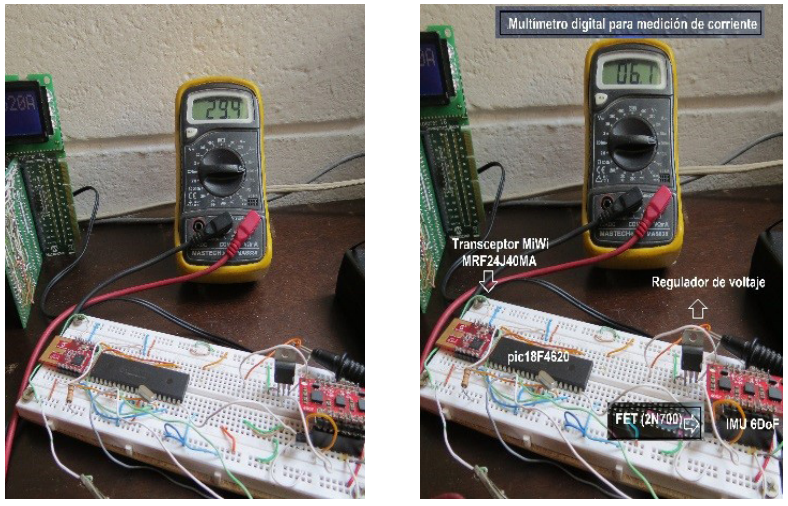

Figure 5. Measurements of sensor node current consumption working in situations described in Table 4 and Table 5 / Medición del consumo de corriente en el nodo sensor en las situaciones ilustradas por las Tablas 4 y 5 
Se obtiene así una disminución significativa en el consumo de corriente del nodo sensor. Efectivamente se comprueba la drástica reducción del consumo de corriente al poner ambos, sensor y transceptor, en modo sleep. Si tenemos en cuenta que la corriente derivada por el regulador de voltaje es de unos $6 \mathrm{~mA}$, entonces el consumo del tándem microcontrolador-transceptor es de $0.1 \mathrm{~mA}$ $(100 \mu \mathrm{A})$, lo que está en mayor consonancia con lo prometido en las hojas de datos de ambos dispositivos.

Algunos reguladores de mayor eficiencia, y también de mayor precio, que la referencia utilizada, podrían bajar el consumo del nodo sensor a unos $2 \mathrm{~mA}$. Es el caso del regulador tipo step-down TSR 1-2433 (Traco Power, 2012) que presenta una cifra cercana a los $2 \mathrm{~mA}$ en modo stand-by.

Las referencias PIC18F4620 y PIC18LF4620 (Microchip Technology Inc., 2004) fueron utilizadas durante las pruebas obteniendo los mismos resultados. Esto se explica ya que los dispositivos LF solo varían su rango de alimentación de voltajes a uno más bajo, de 5.5 a 2 Voltios, pero no sus características de consumo de energía. Los dispositivos designados con LF en su número de parte son más apropiados para aplicaciones alimentadas por batería, porque se garantiza la operación del microcontrolador para voltajes de alimentación más bajos.

La función de MiWi MiAppWriteData (Microchip Technology Inc., 2009) está configurada, originalmente, para enviar un byte (carácter) por transmisión, lo que hace muy ineficiente la gestión de la energía, puesto que, como se conoce, el momento crítico para el consumo de energía corresponde a los periodos de actividad de transmisión del radio transceptor. Para mejorar este aspecto se programó una función que envía cadenas de caracteres, con el fin de reducir el número de transmisiones por mensaje y aprovechar la capacidad de bytes del payload de los paquetes MiWi, que es 127 dado que es un protocolo conforme al estándar IEEE 802.15.4. Si por ejemplo, para enviar los datos de una medición se requieren doce caracteres, el número de transmisiones se incrementa en ese factor si no se usan cadenas, por lo que en 1.200 paquetes transmitidos se enviarían solo los datos correspondientes a 100 mediciones. Con una correcta gestión de las cadenas y el uso de caracteres separadores entre mediciones consecutivas, se podrían enviar unas nueve mediciones por transmisión, lo que resultaría en unas 10.800



Figure 6. Prototype used in experiments / Disposición de elementos utilizada en el experimento the transceiver and microcontroller. If the reader considers that the consumed current from voltage regulator is about $6 \mathrm{~mA}$, consumption of transceiver-microcontroller duo is $0.1 \mathrm{~mA}(100 \mu \mathrm{A})$, datum in accordance with datasheet specifications.

Some high-efficiency regulators (and, subsequently, more expensive) than the one utilized in this work might reduce sensor node consumption up to $2 \mathrm{~mA}$. TSR 1-2433 step-down regulator (Traco Power, 2012) is one of them that introduces a nominal $2 \mathrm{~mA}$ of current in stand-by mode.

References PIC18F4620 and PIC18LF4620 (Microchip Technology Inc., 2004) were used during tests that obtained similar results. This is because Low Frequency $[\mathrm{LF}]$ devices only present variations in nominal voltage supply from $5.5 \mathrm{~V}$ to $2 \mathrm{~V}$, but they do not change their energy consumption features. LF devices specified in their serial number are more suitable for battery-fed applications, since they guarantee microcontroller operation for low supplying voltages.

The MiWi function called MiWiAppWriteData (Microchip Technology Inc., 2009) is factory configured to send one byte (character) per transmission; this is very inefficient in terms of energy consumption, as the reader infers the energy-related critical moment corresponds to activity time in the transceiver. To improve performance in this aspect, I programmed a function that sends strings to reduce the number of transmissions per message and take advantage of payload capacity (127 bytes in total as IEEE 802.15.4 standard suggests). For instance, if the system needs to send 12 characters, the number of transmissions is incremented in a factor of 12 (the number of characters to send) if strings are not used, resulting in sending 1200 packets that represent only 100 measurements. Correct string management and use of separated characters between consecutive measurements leads to 9 measurements per transmission (i.e. 10,800 measurements in 1200 packets). If the system is programmed to send only one measurement per message, the number of transmitted measurements equals the number of packets, as experiments that have been conducted show.

In order to validate changes presented in energy consumption after implementing changes over nodes hardware and software structure, I implemented a network with two nodes, coordinator and sensor node. This prototype is shown in Figure 6. Tests consisted of counting received messages from the coordinator when con- 
tinuous operation is presented, sending packages with measurement data derived from accelerometer's axes in IMU used as sensor.

\section{Results discussion}

After elements are placed as Figure 6 shows, I proceeded to program continuous operation in nodes until package reception ends due to battery depletion; this test was carried out in an internal environment. The display in the coordinator node showed messages indicating the arrival and number of received packages. TABLE 6 summarizes the obtained results for two scenarios previously described in TABLES 4 AND 5.

Obtained results showed an increase up to $62 \%$ more in operation time and transmitted packages in optimal conditions compared with scenario where only firmware measurements to reduce energy consumption are implemented. Experimentation was executed with the same battery and similar charging conditions.

With a couple of Li-Ion batteries and nodes working in optimal conditions, the system was able to send 1032 messages in a 15-hour time period approximately. Afterwards, under the same conditions, I used only one battery and the system managed up to 688 packets in about 10 hours of uninterrupted sensor operation.

Figure 7 shows the implemented hardware/firmware mechanisms in this experimentation searching for improvements in sensor node energy consumption. Some parts of implemented code in microcontroller are shown and generalizations of identified improvement options (from hardware elements until sensor switching) are also displayed.

Adoption of efficient circuital schemes can affect the nodes cost significantly; consequently, this aspect should be considered to implement larger networks with an elevated number of nodes, since cost/benefit analysis must be evaluated for both scenarios. To illustrate this aspect, I explain a hypothetical situation where the system uses a high efficiency and low consumption step- medidas transmitidas en 1.200 paquetes. Si se envía una medición por mensaje, el número de medidas transmitidas será igual al número de paquetes, como es el caso de los experimentos realizados.

Con el fin de validar los cambios que se presentaron en el consumo de energía de los nodos luego de implementar cambios sobre su estructura de hardware y firmware, se procedió a realizar un despliegue de una red de prueba con dos nodos, un nodo coordinador y un nodo sensor. El ensayo consistió en realizar un conteo de los mensajes recibidos por el coordinador al poner a operar el nodo de manera continua, enviando paquetes con los datos de medición derivados de los tres ejes del acelerómetro de la unidad de medición inercial utilizada como sensor.

\section{Discusión de resultados}

Luego de realizar la disposición de los elementos, como se indica en la FiguRa 6, en un ambiente interior, se procede a dejar operar los nodos de manera continua, hasta que no se reciban más paquetes del nodo sensor por agotamiento de la batería. En el display del nodo coordinador se verificó el arribo de los mensajes y se llevó la cuenta de los paquetes recibidos. LA TABLA 6 resume los resultados obtenidos para los dos escenarios mencionados en las TABLAS 4 y 5 .

Table 6. Performance evaluation indicators in optimal conditions (firmware/hardware measurements) and only firmware measurements / Indicadores de desempeño en condición optima (medidas de firmware/hardware) y de medidas solo de firmware

\begin{tabular}{lcc}
\hline \multicolumn{1}{c}{ Indicator / Indicador } & $\begin{array}{c}\text { Switching between IMU and field } \\
\text { effect transistor / Conmutación del sensor IMU } \\
\text { con transistor de efecto de campo }\end{array}$ \\
\cline { 2 - 3 } & Yes / Si & No / No \\
\hline $\begin{array}{l}\text { Transceiver enters sleep mode / Trans- } \\
\text { ceptor entra modo sleep }\end{array}$ & Yes / Si & Yes / Si \\
\hline $\begin{array}{l}\text { Microcontroller enters sleep mode / } \\
\text { Microcontrolador entra en modo sleep }\end{array}$ & Yes / Si & Yes / Si \\
\hline $\begin{array}{l}\text { Number of transmitted packets / } \\
\text { Nümero de paquetes transmitidos }\end{array}$ & 653 & 248 \\
\hline $\begin{array}{l}\text { Approximate operation time (hours) } \\
\text { / Tiempo aproximado de operación (horas) }\end{array}$ & 11 & 4 \\
\hline
\end{tabular}

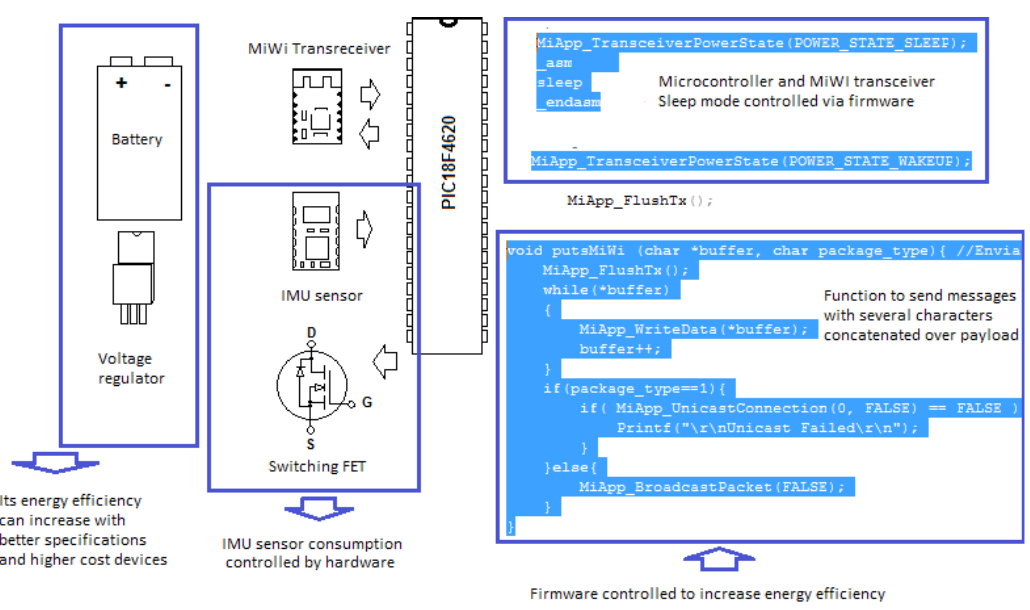

Figure 7. Schematic of hardware/firmware measurements taken in sensor node to increase energy efficiency / Representación esquemática de las medidas a nivel de hardware/firmware tomadas sobre el nodo sensor con el fin de mejorar su desempeño en consumo de energía 
Los resultados obtenidos mostraron un $62 \%$ aproximado de mayor funcionamiento, en cuanto a tiempo y número de paquetes transmitidos, en la condición que consideramos óptima, comparada con la situación en la que solo se toman medidas de firmware para minimizar el consumo de energía del nodo. El experimento se realizó utilizando una misma batería con iguales condiciones de carga.

Con baterías de Li-ion se procedió de la misma manera. Con un par de baterías de este tipo, y el nodo funcionando en condición óptima, se logró enviar 1.032 mensajes en un periodo de 15 horas aproximadamente. Luego, en las mismas condiciones, se utilizó una sola batería de Li-ion (3.7 Voltios), logrando enviar 688 paquetes en un intervalo de alrededor de 10 horas.

La Figura 7 muestra los mecanismos de hardware y firmware implementados en este experimento para mejorar el consumo de energía del nodo sensor. Se muestran algunos apartes del código implementado en el microcontrolador y se indican las generalidades de las opciones de mejora identificadas desde los elementos de hardware, así como la conmutación del sensor.

La adopción de esquemas circuitales eficientes puede influir significativamente sobre los costos de los nodos, un aspecto a tener en cuenta para el despliegue de conjuntos numerosos de nodos, puesto que debe valorarse la relación costo-beneficio para los dos escenarios. Para ilustrar este aspecto veremos lo que sucede si utiliza un regulador step-down de alta eficiencia y bajo consumo. El costo de un regulador de uso común como el LD1117 es de 0.77 dólares en el mercado local de suministro de dispositivos electrónicos. El precio de un regulador de alta eficiencia y bajo consumo de corriente como el TSR 1-2433 (Traco Power, 2012) es de unos 30.5 dólares, también en el mercado local. Tenemos entonces un aumento en el costo de unas 39 veces si se opta por la mejor opción. Este no es un hecho menor y es el diseñador quien debe decidir finalmente qué es lo mejor para su proyecto.

Utilizando el regulador TSR 1-2433 y una batería recargable de NiMH se logró transmitir 1.606 paquetes de manera continua durante un poco más de 24 horas. En la configuración óptima, conmutando el sensor e ingresando en modo sleep periódicamente, se obtuvo un consumo mínimo de $1.32 \mathrm{~mA}$ para los periodos inactivos del nodo y valores que oscilaban entre 9 y 13mA máximo durante el proceso de conversión análoga-digital y transmisión de las mediciones. Se intentó realizar la misma prueba alimentado el nodo con una batería recargable de li-ion de 3.7V, infortunadamente el regulador TSR 1-2433 solo funciona para voltajes de entrada mayores o iguales a 4.75 voltios, lo que imposibilita esta prueba.

El dispositivo electrónico de conmutación elegido, el 2N700 (Fairchild Semiconductor, 1997), presenta un costo de 0.73 dólares en el mercado local y si bien es considerablemente más costoso que sus contrapartes de tipo NPN y PNP (cuesta unos 0,04 dólares por unidad), no incrementa dramáticamente los costos de la circuitería del nodo sensor.

Otro elemento de hardware susceptible de mejora es la batería. En el experimento se utilizaron baterías recargables de tipo $\mathrm{NiMH}$ que cuestan 7.6 dólares en el mercado local y un cargador de 21.7 dólares. En el caso de las baterías tipo LiPo, en el mercado local los down regulator. The price of common regulators such as the LD1117 is $\mathrm{U} \$ 0.77$ in the local market; compared with price of high efficiency and low consumption regulators as TSR 1-2433 (Traco Power, 2012) located between $\mathrm{U} \$ 30$ and $\mathrm{U} \$ 32$ in the local market too, the increase in price between these two products is appreciable. Consequently, the rise in cost is about 39 times if implementation of the most efficient regulator is considered. This is no minor fact and it is the responsibility of the system designer what is better for its project.

Using the TSR 1-2433 regulator and a NiMH rechargeable battery, the system was able to transmit 1606 packets operating continuously during 24 hours approximately. In optimal configuration, i.e. switching the sensor and entering sleep mode periodically, current consumption was $1.32 \mathrm{~mA}$ for inactive periods and values between 9 and $13 \mathrm{~mA}$ during analog/digital conversion and data transmission. I tried to implement a Li-ion battery to feed this proposal, but the TSR regulator only operates for input voltages higher than $4.75 \mathrm{~V}$, making this test impossible.

The switching device chosen in successful tests (2N700 reference) (Fairchild Semiconductor, 1997) presents a Manufacturer's Suggested Retail Price [MSRP] of $\mathrm{U} \$ 0.73$ in the local market; although it is considerably more expensive than other NPN/PNP options (with MSRP only at $U \$ 0.04$ ), choosing the Fairchild $®$ option does not increase costs of circuitry in the sensor nodes dramatically.

Another hardware element with upgrade possibilities is the battery. In this experiment, I used NiMH rechargeable batteries with MSRP of U\$7.6 and a U\$21.6 battery charger, available in local market. For lithium polymer batteries, prices in Colombia are between U\$8 and $\mathrm{U} \$ 21.8$ for batteries, plus $\mathrm{U} \$ 6$ and $\mathrm{U} \$ 35.3$ for chargers. These prices suggest that the equilibrium point between LiPo and NiMH batteries can be found in order to assume battery change in sensor nodes.

Data reliability and stability in wireless communications between sensor node and coordinator was supervised using Wireless Development Studio, traffic analysis tool suitable for MiWi connections. This software operates together with the sniffer node called ZENA (Microchip Technology Inc., 2011). Figure 9 displays detail of data sent from node 3 (sensor node) to node 1 (PAN coordinator). 

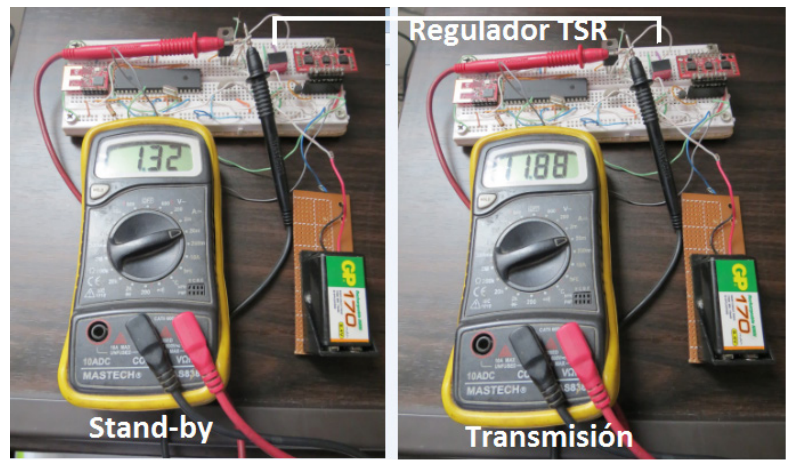

Figure 8. Current consumption measurements during TSR 1-2433 regulator tests / Medición de consumo de corriente (miliamperios) durante prueba con el regulador TSR 1-2433

Extended operation tests were carried out later, where the system dropped a total of 600 packets transmitted and received successfully from the sensor node to coordinator; these tests presented batteries with enhanced charging conditions, non-optimal conditions, and neither switching nor sleep modes in microcontroller. The projection of these results implies an improvement of almost twice in packets processed by the microcontroller in the sleep mode, and an increasing of over 1500 packets with best conditions (i.e. using FET and sleep mode in microcontroller as well as transceiver). This scenario represents more than 24 hours of non-stop data transmission for a single battery charge (NiMH type at $8.4 \mathrm{~V}$ ). It is important to remark that none of the batteries used in this experiment was brand-new; the batteries had experienced several charge cycles since their usage in other WSN projects.

Finally, in another extended test, the sensor was fed with a wet lead-acid battery operating at $12 \mathrm{~V}$. Under this configuration, the system was able to transmit 10800 messages during 150 hours of operation.

\section{Conclusions and future work}

In this work, it was possible to verify some procedures that allow reduction in current consumption of the sensor node under continuous operation time. Through instructions like sleep - recognized for compilers like MPASM and C18 - I was able to force a low-power state in microcontroller that rules sensor node. Also, I verified sleep mode operation in the MiWi MRF24J40MA transceiver through the function MiApp_TransceiverPowerState(), called in the MiApp interface. In a complementary way, the developer can access this low-consumption state through



Figure 9. Verification of packets reception during tests / Verificación de la recepción de paquetes durante las pruebas

precios de baterías recargables de 3.7 oscilan entre unos 8 y 21.8 dólares, y sus cargadores entre 6 y 35.3 dólares. Estos costos sugieren que puede encontrarse un punto de equilibrio para asumir el cambio de baterías en el nodo sensor.

La integridad de los datos y la continuidad de las comunicaciones entre el nodo sensor y el coordinador se superviso con la herramienta de análisis de tráfico MiWi llamada Wireless Development Studio, la cual trabaja en conjunto con el nodo tipo sniffer llamado ZENA (Microchip Technology Inc., 2011). En la Figura 9 se muestra el detalle de los datos enviados desde el nodo 3 (nodo sensor) hacia el nodo 1 (coordinador de la PAN).

En pruebas de operación extendida posteriores se obtuvieron cifras cercanas a los 600 paquetes transmitidos y recibidos exitosamente, desde el nodo sensor hacia el nodo coordinador, cuando se tenían unas baterías con mejores condiciones de carga y las condiciones menos óptimas de consumo de energía en el nodo, sin conmutación ni modo sleep en el microcontrolador. La proyección de estos resultados indica una mejora que resultaría en casi el doble de paquetes con el microcontrolador ingresando al modo sleep, y en más de 1500 paquetes para las mejores condiciones, es decir, con el FET y radio con sleep al igual que el microcontrolador. Esta proyección indicaría más de 24 horas de envío continuo de paquetes para una sola carga de batería tipo NiMH de $8.4 \mathrm{~V}$. Es importante anotar que ninguna de las baterías utilizadas era nueva y que estas ya habían experimentado numerosos ciclos de carga, debido a su uso en otros experimentos alimentando nodos en redes inalámbricas de sensores.

Finalmente, vale la pena anotar que en una prueba de operación extendida se alimentó el nodo sensor con una batería recargable, de tipo seco, de plomo-ácido a 12 Voltios y con ella se logró la transmisión de 10.800 mensajes, durante 150 horas de operación.

\section{Conclusiones y trabajo futuro}

En este trabajo fue posible verificar algunos procedimientos que permiten la reducción del consumo de corriente de un nodo sensor sometido a pruebas de trabajo continuo durante horas. A través de instrucciones como sleep, reconocidas por compiladores -como MPASM y C18- se pudo poner en estado de bajo consumo al microcontrolador que gobernaba al nodo sensor. Igualmente, se verificó el funcionamiento del modo sleep 
en el transceptor MiWi MRF24J40MA mediante la llamada a función MiApp_TransceiverPowerState( ) de la interfaz MiApp de Microchip. De manera complementaria, el desarrollador puede acceder a este estado de bajo consumo del transceptor a través de la llamada a función MiMAG_PowerState( ), pero de manera no tan transparente como en el primer caso.

A partir de los resultados obtenidos se infiere que, para verificar el supuesto de bajo consumo que deben tener los nodos sensores en redes inalámbricas de operación sobre periodos extendidos de tiempo, es necesario tomar un enfoque que plantea intervenciones, tanto a nivel de firmware, desde el código implementado en el microcontrolador, como de hardware, es decir a nivel circuital.

Se encontró que la utilización en el circuito del nodo sensor de dispositivos de mejores especificaciones, para el caso de las baterías y del conversor DC-DC, y de precio más elevado, puede resultar en una mejora de la eficiencia en términos de consumo de energía. Sin embargo, la balanza costo/beneficio puede inclinarse hasta un punto de no retorno que derive en poca o ninguna viabilidad económica para el despliegue de redes inalámbricas con muchas unidades de nodos sensores.

A partir de la valoración del desempeño de nodos sensores desplegados en diferentes aplicaciones y en ambientes exteriores (outdoor) e interiores (indoor), se podrían establecer algunos indicadores acerca de la relación costo-beneficio en relación con la adopción de medidas de firmware, hardware o de ambos tipos, al buscar reducir el consumo de energía en redes MiWi.

Algunas consideraciones previas indican que las mayores dificultades de optimización podrían enfrentarse con redes desplegadas en exteriores, en aplicaciones en entornos no urbanos, industriales o sobre obras de infraestructura, dado que las condiciones de instalación y las especificaciones de los sensores con la robustez requerida para estos casos, en muchas ocasiones, aún no están concebidas para trabajo con bajos niveles de corriente y de voltaje. Adicionalmente, la conmutación de cargas se hace más compleja a medida que sus especificaciones eléctricas cambian. $\$ \&$ function MiMAC_PowerState(), but in a manner not as transparent as the first case.

According to the obtained results, the reader infers that, in order to verify the assumption related with low consumption that sensor nodes should have in wireless networks in long operation times, it is necessary to follow approaches that present interventions, both at firmware level (e.g. implemented code in microcontroller) and hardware level (i.e. circuitry).

The results showed that utilizing devices with better specifications in sensor node circuits lead to increments in efficiency in terms of energy consumption. Nevertheless, the cost/benefit balance can be inclined towards a non-return point, resulting in low or even absence of economic profitability for projects with high number of sensor nodes.

From performance evaluation of sensor nodes implementing several applications and located in outdoors and indoors, some indicators regarding cost/benefit relationship can be established. These indicators are related to adoption of measurements in firmware, hardware, or both, following the reduction of energy consumption in MiWi networks.

Some previous considerations indicate that greater optimization difficulties might be faced implementing outdoor networks, in applications in urban or industrial environments. This because installation conditions and sensor specifications related with robustness are required in these environments; in many cases, these sensors are not conceived for operation with low voltage and current levels. Additionally, switching loads is more complex if electric specifications in sensors change. ST

\section{References / Referencias}

Burns, A., Greene, B. R., McGrath, M. J., O'Shea, T. J., Kuris, B., Ayer, S. M, Cionca, V. (2010). SHIMMERTM - A wireless sensor platform for noninvasive biomedical research. IEEE Sensors Journal, 10(9), 1527-1534. doi:10.1109/JSEN.2010.2045498

Fairchild Semiconductor. (1997). 2N7000 N-Channel Enhancement Mode Field Effect Transistor. Retrieved from: https://www.fairchildsemi.com/products/discretes/fets/mosfets/2N7000.html

Fraser, M., Elgamal, A., He, X., \& Conte, J. (2009). Sensor network for structural health monitoring of a highway bridge. Journal of Computing in Civil, 24(1), 11-24. Available at http://ascelibrary.org/doi/abs/10.1061/ (ASCE)CP.1943-5487.0000005

Gargiulo, M., Guerriero, P., Daliento, S., Irace, A., D’Alessandro, V., Crisci, M., Smarrelli, M. (2010). A novel wireless self-powered microcontroller-based monitoring circuit for photovoltaic panels in grid-connected systems. In SPEEDAM 2010 - International Symposium on Power Electronics, Electrical Drives, Automation and Motion (pp.164-168). doi:10.1109/SPEEDAM.2010.5542234

Microchip Technology Inc. (2004). PIC18F2525/2620/4525/4620 Data sheet enhanced flash microcontrollers with 10-Bit A / D and nanowatt technology. Retrieved from: http://www.tme.eu/es/Document/367303b6b4e89b4a2fc1a86def563d0b/PIC18F4620-I P.pdf 
Microchip Technology Inc. (2008). MRF24J40MA data sheet. Retrieved from: http://ww1.microchip.com/downloads/en/DeviceDoc/70329b.pdf

Microchip Technology Inc. (2009). Microchip wireless (MiWi) application programming interface - MiApp. Retrieved from: http://www.microchip.com/wwwAppNotes/AppNotes.aspx?appnote =en544039

Microchip Technology Inc. (2010a). Microchip MiWi P2P wireless protocol. Retrieved from: http://ww1.microchip.com/downloads/en/AppNotes/01204B.pdf

Microchip Technology Inc. (2010b). Microchip MiWi wireless networking protocol stack. Retrieved from http:// ww1.microchip.com/downloads/en/AppNotes/AN1066\%20-\%20MiWi\%20App\%20Note.pdf

Microchip Technology Inc. (2011). ZENA TM wireless adapter user's guide. Retrieved from http://www. microchip.com/DevelopmentTools/ProductDetails.aspx?PartNO=AC182015-1

Microchip Technology. (2009). Microchip wireless (MiWiTM) media access controller - MiMAC. Retrieved from: http://www.microchip.com/wwwAppNotes/AppNotes.aspx?appnote=en544032

Otniel Portillo-Rodríguez, Jessica C. Alcaide-Barragán, Oscar O. Sandoval González, Adriana H. Vilchis-Gonzalez, Juan Carlos Ávila-Vilchis, M. R.-H. (2011). A framework to develop reliable low cost wireless sensors networks using the IEEE 802.15.4 standard [paper in IEEE 22a Reunión Internacional de Otoño, de Comunicaciones, Computación, Electrónica, Automatización, Robótica y Exposición Industrial ROC\&C'2011, Acapulco. (pp. 5-10).

Pannila, E., Tuominen, A., \& Edirisinghe, M. (2011). Gas sensor data collection over an RF network. In Proceedings of the Technical Sessions - IPSL, 27, 92-99. Colombo, Sri Lanka: Institute of Physics Sri Lanka.

Powering microcontrollers with scavenged energy | Digikey. (2012, 08/22). Retrieved from: http://www.digikey. com/es/articles/techzone/2012/aug/powering-microcontrollers-with-scavenged-energy

ST. (2013). LD1117 Adjustable and Fixed Low-Dropout Voltage Regulator. Retrieved from: http://www.ti.com/lit/ ds/symlink/tlv1117-50.pdf

Traco Power. (2012). DC / DC converters TSR-1 series. Retrieved from: http://www.tracopower.com/products/ tsr1sm.pdf 


\section{CURRICULUM VITAE}

Gustavo Meneses Benavides Received his Electrical Engineering degree from Universidad Nacional de Colombia (Medellín, 2001), and his MSc in Engineering from the Universidad de Antioquia. He is professor and researcher in Universidad de San Buenaventura, Medellín. His current research interests are telemetry systems, wireless sensor networks and structural health monitoring. He is an IEEE member. / Ingeniero Eléctrico de la Universidad Nacional (Medellín, Colombia); Especialista en Automatización Industrial de la Universidad de Antioquia; Máster en Ingeniería, área Electrónica de la Universidad de Antioquia (Medellín). Actualmente es docente e investigador de la Universidad de San Buenaventura, sede Medellín. Sus áreas de interés son la telemetría, la instrumentación electrónica y las telecomunicaciones. 